\title{
Indirect effects of peri- and postnatal choline treatment on place-learning abilities in rat
}

\author{
FRANÇOISE SCHENK and CATHERINE BRANDNER \\ Institut de Physiologie, Lausanne, Switzerland
}

\begin{abstract}
This work was aimed at analyzing the effects of perinatal choline supplementation on the development of spatial abilities and upon adult performance. Choline supplementation $(3.5 \mathrm{~g} / \mathrm{L}$ in $0.02 \mathrm{M}$ saccharin solution in tap water) was maintained for two weeks before birth and for up to four weeks postnatally. Additional supplementation was maintained from the fifth to the tenth week postnatally. Spatial-learning capacities were studied at the ages of 26,65 , or 80 days in a circular swimming pool (Morris place-navigation task) and at the age of 7 months in a homing arena. Treatment effects were found in both juvenile and adult rats, and thus persisted for several months after the cessation of the supplementation. The choline supplementation improved the performance in the water maze in a very selective manner. The most consistent effect was a reduction in the latency to reach a cued platform at a fixed position in space, whereas the improvement was limited when the platform was invisible and had to be located relative to distant cues only. However, after removal of the goal cue, the treated rats showed a better retention of the training position than did the control rats. A similar effect was observed in a dry-land task conducted in the homing arena. The choline supplementation thus induced a significant improvement of spatial memory. But since this effect was only evident following training with a salient cue, it might be regarded as an indirect effect promoted by an optimal combination of cue guidance with a place strategy.
\end{abstract}

The metabolisms both of membrane phospholipids and of acetylcholine require choline as a precursor. It is therefore of prime importance to assess whether the long-term stability of the cholinergic system can be affected by choline uptake during early development and whether it is accompanied by changes in learning and memory capacity. A chronic choline supplementation during the gestation and postnatal periods has led to longlasting changes in neurochemical and behavioral parameters in adult rats, long after the cessation of the treatment. The improved spatial performance was assessed in a radial maze and was correlated with a decrease in cholineacetyltransferase (ChAT) level in the hippocampus, but not in the cortex, and with an increase in muscarinic receptor density in the cortex, but not in the hippocampus (Meck, Smith, \& Williams, 1989). A similar perinatal treatment affected also the morphology and distribution of cholinergic neurones in the basal forebrain (Loy, Heyer, Miller, \& Linder, 1992; Loy, Heyer, Williams, \& Meck, 1991). A shorter dietary treatment (embryonic age 12-17 days) led to significant reductions in ChAT in the hippocampus and in the striatum about 35 days postnatally (Jackson, Meck, Williams, \& Blusztajn,

This research was supported by Grant 31.30068 .90 from the Fonds National Suisse de la Recherche Scientifique. We are grateful to M. Gafner for assistance with animal training. We thank Hoffmann La Roche (Basel) for providing us with female breeders of the PVG strain. Correspondence should be addressed to F. Schenk, Institut de Physiologie, Bugnon 7, 1005 Lausanne, Switzerland (e-mail: fschenk@ ul9000.unil.ch).
1992). These authors also reported neurochemical changes 90 days postnatally, as expressed by an overall reduction in ChAT activity, without significant effect in any particular brain region. A similar treatment (ED 12 to birth) advanced the postnatal development of place learning in the Morris navigation task by 3 days and reduced age-related changes in spatial working memory in 21-month-old rats (Dallal, Meck, \& Williams, 1992). A reduction of age-induced memory impairment was still evident in 26-month-old rats trained in a radial-maze task (Meck \& Williams, 1992). Finally, some effects of this treatment were also observed on general measures of exploratory behavior (Tees \& Johnston, 1992).

With one exception (Dallal et al., 1992), the improvements in spatial abilities were assessed in a radial-maze task, which seems particularly sensitive to a change in central cholinergic activity. For example, selective lesions of the medial septal nucleus that reduced the cholinergic innervation of the hippocampus impaired radial-maze task performance but not place learning in the Morris task (Decker, Radek, Majchrzak, \& Anderson, 1992). Sinden, Hodges, and Gray (1995) also reported that a cholinergic deficit due to lesions of the medial septum or of the nucleus basalis affected the performance in a radial-maze task more severely than in a place-learning task. However, if training is conducted with a salient cue indicating the goal position, but the memory of the training place is assessed after removal of the salient cue, the task is particularly sensitive to septal lesions, aging, or immaturity (Schenk, 1985; Schenk, Contant, \& Werffeli, 1990; Schenk, Grobéty, Lavenex, \& 
Lipp, 1995). The capacity to learn a spatial position in the presence of an overshadowing local cue might thus depend on a central cholinergic function.

This short review of the behavioral effects of perinatal choline supplementation indicates that the radial-maze task is particularly sensitive to choline supplementation, while the effect on a place-learning task was mainly to advance postnatal performance. We have thus decided to analyze more qualitatively exactly what changes in visuospatial capacities are induced by a perinatal choline treatment, and the present series of experiments was aimed at establishing how it might affect various aspects of place-learning capacities in a Morris navigation task and in a homing arena (Schenk, 1989). The prenatal treatment was administered as described in Meck et al. (1989). The postnatal treatment was continued through the mothers' drinking water until the end of the fourth week postnatal, and was then provided as an individual supplementation until the end of the second month of life. This procedure was preferred to intragastric cannulation, to allow postnatal treatment without inducing any particular stress that might interfere with direct choline effects.

Nine adult females of the PVG strain provided by Hoffmann-La Roche Basel were exposed to a $0(n=6)$ or $5 \mathrm{ml} / \mathrm{L}$ solution of $70 \%$ choline chloride $(n=4)$, in a saccharine solution $(1.2 \mathrm{~g} / \mathrm{L})$, as described in Meck et al. (1989). The choline supplementation started at the end of the first week of pregnancy, and was maintained until after weaning. All the females were kept separate in standard macrolon cages $(38 \mathrm{~cm}$ wide $\times 60 \mathrm{~cm}$ long $\times$ $20 \mathrm{~cm}$ high) provided with a wooden nest box and cellulose as nesting material. The litter size varied between 8 and 12 in both the choline-treated and control groups.

During gestation, the treated females consumed a mean of $31 \mathrm{ml} /$ day, and the control females $29 \mathrm{ml} /$ day. This supplementation was maintained until four weeks after birth. The total amount of water consumed by the litters was about $80 \mathrm{ml} /$ day at the end of the third postnatal week. From Day 29 onward, the young rats were provided daily with choline in diluted condensed milk $(0$ or $0.5 \mathrm{ml}$ of $70 \%$ choline solution), to provide a supplementary daily intake of about $40 \mathrm{mg}$ choline per day. This treatment was progressively discontinued from postnatal Day 65 over 10 days. We observed no difference in weight or gross morphology between the cholinetreated and control individuals.

\section{EXPERIMENT 1}

This experiment was aimed at assessing the effects of peri- and postnatal choline supplementation on spatiallearning abilities in a Morris place-navigation task. The performances of immature subjects ( 26 days postnatal) and adult subjects ( 80 days postnatal) were studied in order to detect the possible effects of this treatment on the maturation of cognitive brain functions. Two different training conditions were used in order to assess the capacity to rely on distant landmarks in the presence or in the absence of a salient cue associated with the goal. To this end, half of the subjects were trained using the standard place-navigation paradigm (i.e., they were trained to escape onto an invisible platform at a fixed location relative to distant room cues). For the other half of the subjects, this location was simultaneously signaled by a salient cue suspended above it.

\section{Method}

Subjects and treatment. Twenty rats from two different control litters and 16 rats from two choline-treated litters served as subjects. The litters were kept with the mothers until the age of 42 days. The rats were then kept in groups of 4-5 individuals of the same sex in standard macrolon cages $(38 \mathrm{~cm}$ wide $\times 60 \mathrm{~cm}$ long $\times 20 \mathrm{~cm}$ high) with a wooden nest box, and had ad lib access to food and water.

Apparatus. A large circular water tank (diam $=160 \mathrm{~cm}$, wall height $=60 \mathrm{~cm})$ painted white was filled with water $\left(25^{\circ} \mathrm{C}\right)$ and milk $(0.5 \mathrm{~L})$ to a depth of $30 \mathrm{~cm}$. This pool was located in a room containing several uncontrolled cues (door, posters, suspended dark cloth) at a minimum distance of $100 \mathrm{~cm}$ from the pool wall. Four orthogonal starting positions were spaced around the perimeter of the pool, dividing its surface into four quadrants. An invisible escape platform made of transparent Plexiglas (diam $=9 \mathrm{~cm}$ ) could be placed in the center of each quadrant. A black cylinder (diam $=4.5 \mathrm{~cm}$, length $=10 \mathrm{~cm}$ ) was suspended $30 \mathrm{~cm}$ above the platform for a cuing procedure. A video camera was placed directly above the center of the pool.

For activity recording, a small plastic cage $(40 \mathrm{~cm}$ wide $\times 26 \mathrm{~cm}$ long $\times 26 \mathrm{~cm}$ high) was placed in the center of a large circular wooden board, on top of the water maze. Access to this open field was made possible through a small circular aperture $($ diam $=5 \mathrm{~cm})$ in the lower part of one wall of the cage

Behavioral procedure. Control and choline-treated subjects were assigned to experimental groups trained in two different conditions. In one condition ( lace only), the invisible plat form could be located relative to distant room cues only; in the other condition (place and cue), the position of the platform was also signaled by a dark cylinder suspended above it. All of the subjects received 30 trials over 4 days (i.e., $4,10,12$, and 4 trials, respectively). A probe trial, during which the rat was left in the pool for $60 \mathrm{sec}$ without platform or cue, was conducted on Day 3, following Trial 22.

Half of the subjects were trained as juveniles, from the age of 26 days-namely, 10 control rats (6 males and 4 females) and 8 choline-treated rats ( 4 males and 4 females), with half of the groups being trained in the place-only condition, and the other half in the place and cue condition. The remaining $10 \mathrm{cont}$ rol $(5$ males and 5 females) and 8 choline-treated subjects ( 5 males and $3 \mathrm{fe}$ males) were trained at the age of 80 days, half of the groups in the place-only and the other half in the place and cue condition. At this time, the subjects first trained at the age of 26 days were retrained following the same procedure, with each subject being retested with the same platform position and cue condition.

For an activity test, each subject was left in the closed plastic cage for $30 \mathrm{~min}$, after which the circular door was opened and the rat was free to explore the circular open field (diam $=160 \mathrm{~cm}$. with no wall) for $10 \mathrm{~min}$. All of the juvenile rats were tested individually at the age of 39 days.

Data analysis. During training in the water maze, each trial was video recorded and the escape latency was measured. As is usually the case with latency data, the standard deviation varied as the mean latency, so that a logarithm transformation was operated on the original data to avoid biases in the analyses of variance (ANOVAs; Snedecor \& Cochran, 1971). Eight trials (Trials 19-26) and the probe trial were analyzed by an XY video tracker (Kukam S.A., 
Lausanne), which allowed the path length and time spent in different parts of the pool to be measured. However, some of the juvenile rats did not provide enough contrast on the video image, so that their trials had to be analyzed manually, as described in Schenk (1985).

For the activity tests, the sequence was video recorded. The time taken to leave the small cage to enter the open field and the number of movements in and out of the small cage (i.e., the number of exploratory bouts in the open field separated by a return to the small cage) were registered for $10 \mathrm{~min}$.

\section{Results}

Spatial water-maze task. In this experiment, we did not detect any significant effect of sex; the results shown by males and females were therefore grouped for further analyses. The choline-treated rats did not show any particular detectable behavioral abnormality. The effects of the treatment on the acquisition of the spatial task by naive rats are shown in Figures $1 \mathrm{a}$ and $\mathrm{b}$. A four-way ANOVA (treatment. age, training condition, and blocks of trials) of the latencies revealed significant effects of treatment $[F(1,24)=4.82, p=.038]$, training condition $[F(1,24)=29.53, p=.0001]$, and age $[F(1,24)=27.27$, $p=.004]$. There was also clear evidence of learning in all the groups [block effect, $F(6,144)=60.62, p=$ $.0001]$. A significant interaction between treatment, training condition, and block $[F(6,144)=2.78, p=$ $.014]$ indicated that the effect of the treatment was more evident during the second part of training and in the cued condition.

Separate three-way ANOVAs (treatment, age, and block effects) that were conducted for both conditions indicated that the treatment effect was not significant in the place-only condition $[F(1,12)=.73$, n.s. $]$ and did not interfere with other factors. In the place and cue condition, the treatment effect was significant $[F(1,12)=$ $9.59, p=.009]$, and there was an interaction between block and treatment effects $[F(6,72)=4.63, p=.0005]$. Indeed, the treatment effect was only significant from Block 4 onward $(p<.02)$.

Two different analyses were conducted in order to assess the efficacy of escape paths. First, we recorded the frequency of escape trials with a maximal latency of $3 \mathrm{sec}$, which, in our situation, indicates an almost straight escape path. As expected, the treatment effect was highly significant in the place and cue condition [twoway ANOVA: treatment, $F(1,13)=53.57, p=.001$; age, $F(1,13)=12.43, p=.004]$, without interaction. In the place-only condition, there was also a treatment effect increasing the frequency of short escape trials in the experimental groups $[F(1,15)=5.53, p=.03]$. A nearly significant interaction between treatment and age $[F(1,15)=3.83, p=.069]$ indicated that, in this condition, the treatment increased the frequency of rapid escape trials in the adult rats [one-way ANOVA: $F(1,8)=$ $6.59, p=.03$ ], but did not do so in the juvenile subjects.

Escape efficacy was also assessed from the distance swum in Trials 27-30 (Block 7). The shorter escape latencies recorded in the treated groups were due to shorter escape paths. Separate two-way ANOVAs (treatment and age) confirmed that there was a significant effect of treatment on swim distances, with both juvenile and adult choline-treated groups trained in the place and cue condition having a shorter escape-path length than their control groups [one-way ANOVAs: treatment effect- juveniles, $F(1,7)=60.0, p=.0001$; adults, $F(1,7)=6.15$, $p=.048]$. In addition, there was a significant effect of treatment in the adult group trained in the place-only condition $[F(1,8)=5.91, p=.041]$.

The rats first trained when juveniles, at the age of 26 days, were later retrained when 80 days old. Figure $1 \mathrm{c}$ shows that the effect of the treatment was still clearly marked in the cued condition at the end of the training period (Block 7). A two-way ANOVA (treatment and training condition) of the latencies in Block 7 revealed a highly significant effect of the training condition $[F(1,14)=$ $35.57, p=.0001]$ and an interaction between treatment effect and training condition $[F(1,14)=8.93, p=$ .0098 ], indicating that the treatment effect was only evident in the cued condition (Fisher post hoc analyses). A comparison of the latencies of the retrained rats with those of the naive rats (three-way ANOVA: treatment, training condition, and test age) showed a significant effect of experience $[F(1,28)=32.07, p=.0001]$.

During the $60-\mathrm{sec}$ probe trial in which the rats were left in the pool without platform or cue, all the groups trained in the place-only condition showed a significant discrimination of the training sector. In the place and cue training condition, only the adult rats showed a significant discrimination of the training sector. A three-way ANOVA (treatment, age, and training condition) of the time spent in the training sector revealed a significant effect of condition $[F(1,28)=11.44 . p=.0021]$ and age $[F(1,28)=23.85, p=.0001]$, and an interaction between these factors $[F(1,28)=16.31, p=.0004]$. Neither the overall effect of the treatment $[F(1,28)=3.75$, $p=.063]$ nor the interaction between training condition and treatment $[F(1,28)=3.99, p=.055]$ reached significance. Separate two-way ANOVAs (age and training condition) were conducted in both treatment groups due to the highly significant interactions between age and training condition. A significant interaction between age and condition was obtained in the control groups $[F(1,16)=5.09, p=.038]$ and in the choline-treated groups $[F(1,12)=10.33, p=.007]$, indicating that the discrimination of the training quadrant was lower in both groups of immature rats trained in the cued condition. Separate two-way ANOVAs (treatment and training condition) were then conducted in both age groups. As suggested by Figure 2, there was an important effect of the training condition in the juvenile rats $[F(1,14)=17.67$. $p=.0009$ ], without treatment or condition $\times$ treatment effect. In contrast, the adults showed a significant effect of treatment $[F(1,14)=6.28, p=.025]$ and of treatment $\times$ condition $[F(1,14)=12.89, p=.003]$. Post hoc analyses were conducted with the Mann-Whitney test, revealing that the group of adult choline-treated rats trained in the cued condition had a more pronounced bias toward the training quadrant than had either the con- 
a) 26 days

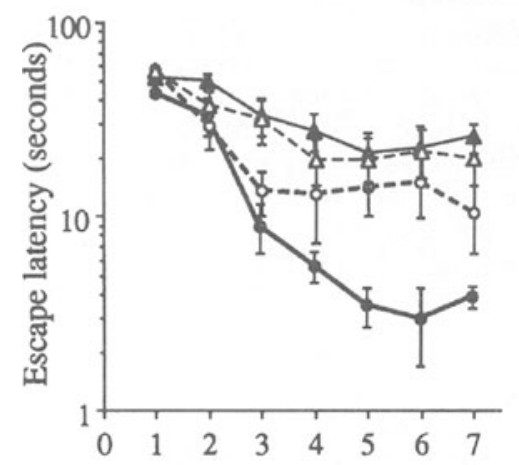

b) 80 days

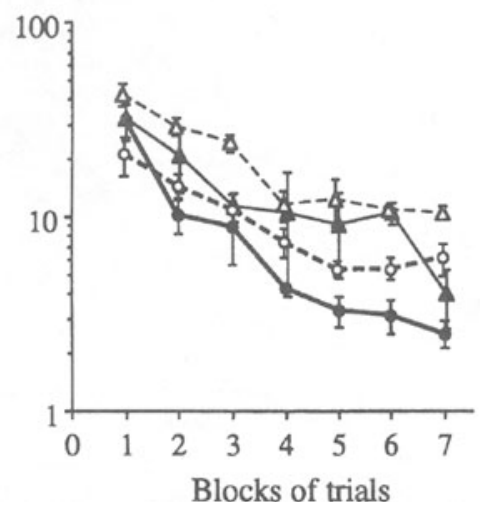

c) 80 days retrained

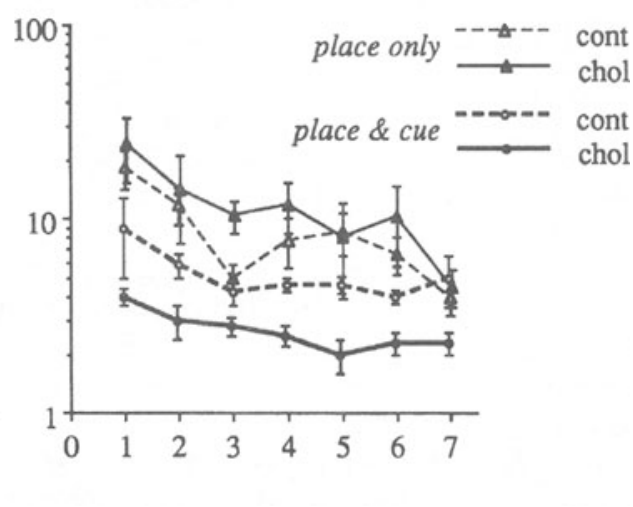

Figure 1. Mean ( $₫ S E M$ ) escape latencies (logarithmic scale) during training in the invisible-platform (place-only) and in the cued-platform (place-and-cue) water-maze task for rats with choline supplementation (choline) and for control rats at different times postnatally (a) naive, 26 days old; (b) naive, 80 days old; (c) retrained, 80 days old. Blocks 1 and $4-7$ consisted of four consecutive trials; Blocks 2 and 3 consisted of five consecutive trials (see behavioral procedure).

trol group trained in the same condition $(z=2.31, p=$ $.021)$ or the treated group trained in the place-only condition $(z=2.18, p=.029)$.

Activity measure. The number of exploratory bouts into the open arena was higher in the choline-treated subjects and in the females. Both effects were equally significant in a combined two-way ANOVA $[F(1,32)=$ $4.77, p=.036]$, but no separate between-group comparison reached significance (male control group, mean \pm $S E M$ of the number of exploratory bursts $=2.9 \pm .5$; male choline-treated group $=4.3 \pm .9$; female control group $=4.3 \pm .5$; female choline-treated group $=6.1 \pm 1.1$ )

\section{Discussion}

In this experiment, the rats aged 26 days at the start of training were clearly impaired in both conditions as compared with the young adults. The slower acquisition of the immature rats and the pronounced overshadowing effect of the goal cue in this age group confirm earlier results that 4-week-old rats are impaired in the acquisition of the Morris task and show specific impairment during a probe trial following training with a salient proximal cue (Chevalley \& Schenk, 1987; Schenk, 1985).

The choline supplementation improved the performance of juveniles and adults trained in the place-andcue condition, but had only a marginal effect when the rats were trained in the place-only condition. In the presence of the goal cue, both juvenile and adult cholinetreated rats had shorter escape latencies than the controls. In addition, the adult choline-treated rats had also a stronger bias toward the training quadrant following removal of the suspended cue, while there was no such effect in the immature choline-treated subjects.

Thus, instead of improving place learning per se, the treatment with choline seemed to predominantly affect the probability that the rats would head toward a salient landmark such as the suspended cue, or, possibly, toward more distant landmarks behind the escape platform. Path length was also significantly decreased. A close examination of the escape paths suggested that there was a high frequency of very short trajectories in the treated adults in both conditions. In the cued condition, this tendency was accompanied by a marked reduction of both distance and latency because it could ensure rapid landing on the cued platform. When the platform was not cued, it was often missed by a few centimeters only, which resulted in prolonged latencies. The treated rats trained in the place-only condition had thus a mixed population of short and long escape paths, which attenuated the between-group difference in mean latency.

Juvenile rats were still receiving choline supplementation at the time of training, while the 80-day-old subjects had been weaned 5 days before. This suggests that age and treatment effects might be confounded, since the oldest rats were no longer receiving concurrent activa-

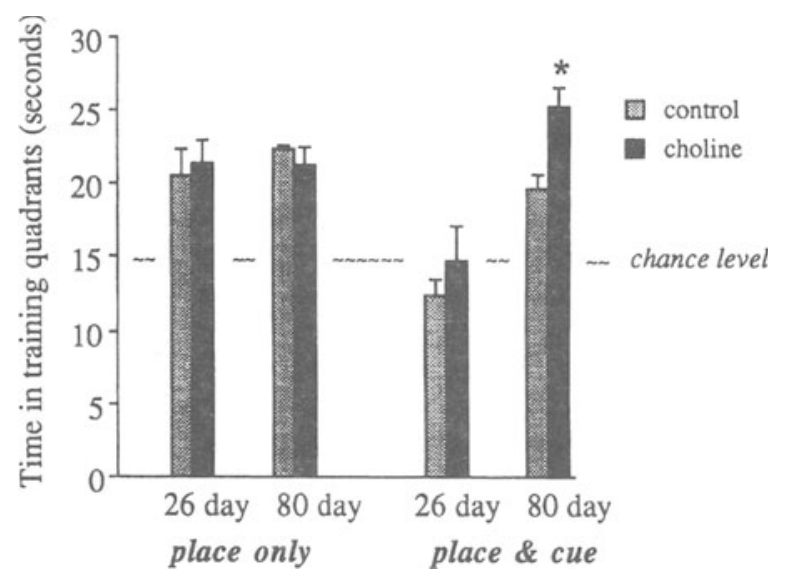

Figure 2. Mean time ( $\pm S E M)$ spent in the training quadrant during a 60-sec probe trial (no platform or proximal cue present) following 22 training trials in the place-only or in the place-and-cue condition. *Significant difference $(p<.05)$ between the choline-treated and control groups. 
tional choline. Both age groups demonstrated a similar improvement in escape latencies in the cued condition, while only the oldest showed an improvement in quadrant time. Thus, one cannot discard the possibility that the acute choline treatment was preventing the quadrant effect. But at the same time, this indicates that neither a combined treatment in the immature rats nor a mere organizational effect of the choline in the adult rats led to a systematic reduction in escape latency during place training.

This experiment thus suggests that the choline treatment had mainly indirect effects on spatial memory, as such effects were only evident in the cued condition. In order to better characterize this behavioral effect, two experiments were undertaken, first, to establish whether the benefit of the choline treatment might also be evident in a nonspatial task, and second, to determine whether the treatment might improve place memory on solid ground, on which ballistic trajectories were less probable.

\section{EXPERIMENT 2}

The better performance of the choline-treated rats in Experiment 1 suggests that they were more rapid at detecting any salient cue. This might have been due to a better memory of the platform position that facilitated a rapid orientation toward the cue upon release in the pool. But it might also have been due to an improved capacity in detecting any salient cue. Experiment 2 was designed to assess whether the choline treatment might improve a general attention capacity so that rats would also be more rapid to detect a salient cue at an unpredictable position in space. Moreover, a distractor cue was introduced in a second training phase to assess distractibility and cuediscrimination capacity, which might be related to selective attention capacity. It was assumed that a sudden immersion into the water at start positions might force the subjects to detect the cue while already swimming, which might prevent an optimal orientation toward the cue until the rats actually faced it. A special starting procedure was therefore designed to allow the rats enough time to detect the cue before starting to swim. The effect of increased attention capacity might thus be more evident when the subjects are allowed to orient toward the cue before being immersed.

\section{Method}

Subjects. Nineteen rats from three different control litters $(10$ females and 9 males) and 20 rats from two choline-treated litters ( 13 males and 7 females) served as subjects. They were maintained as in Experiment 1. They were tested at the age of 65 days.

Apparatus. The same water tank was used for this second experiment. Either a black cylinder $($ diam $=4.5 \mathrm{~cm}$, length $=10 \mathrm{~cm})$ or a white tennis ball covered by a black disk (diam $=12 \mathrm{~cm}$ ) was suspended $30 \mathrm{~cm}$ above the platform for a cuing procedure. A starting platform made of a plastic grid held by two metal wires could be suspended on the tank wall at the usual starting positions. When placed on this platform, a rat was at water level and could not climb back.

Behavioral procedure. Training was conducted in two consecutive phases. In Phase 1, the rats were given 28 trials over 4 days $(8,8,8$, and 4 trials, respectively), with the cued platform at one of four possible positions in the tank, in the center of one of the quadrants. There were four possible starting positions along the wall, in the center of each quadrant, but the escape platform was never placed immediately facing a starting position. Different platform and starting positions were used on each trial on a pseudorandom schedule, such that each starting point was used twice within the 8 daily trials. Moreover, the relative locations of the starting point and platform on each trial were arranged so as to avoid more than two identical (right or left) motor responses in sequence. The black cylinder cue was used for half of the subjects in each treatment condition, and the tennis ball served as a cue for the remaining subjects. In addition, half of the subjects in each group were introduced into the water by hand (immediate usual starting procedure), whereas the other half were allowed to wait for $15 \mathrm{sec}$ on the starting platform (delayed start). They were then forced into the water by lowering the platform, which was then removed from the tank.

At the end of the fourth daily training session, all the rats were given a special probe trial, during which they were allowed to swim in the pool in the absence of the escape platform, but with the cue suspended in the center of a quadrant.

In Phase 2, starting on Day 5 , the rats were further trained with an additional irrelevant cue (i.e., the tennis ball or the black cylinder, depending on which one had been used as the relevant cue during initial training) suspended in an empty quadrant, adjacent or opposite to the quadrant with the escape platform. For each trial, the distractor-cue position was chosen to represent an alternative unreinforced escape position at the same distance from the start as the correct reinforced cue. They were given 20 such trials over three days $(8,8$, and 4 trials, respectively).

Nine control rats ( 4 males and 5 females) and 10 choline-treated rats ( 6 males and 4 females) were trained with the immediate-start procedure, whereas 10 control rats ( 5 males and five females) and 10 choline-treated rats ( 7 males and 3 females) were trained with the delayed-start procedure.

\section{Results}

Training with a single cue (Phase 1 ). There were no significant sex differences in the separate analyses of the latencies: the results of males and females were therefore combined for further analyses. Figure 3 a shows that all the groups learned to escape onto the cued platform during the first phase of training [block effect, $F(6,192)=94.08, p=.0001]$. This three-way ANOVA (treatment, starting position, and block) revealed also a significant effect of treatment $[F(1,32)=5.43, p=$ $.026]$ and of starting condition $[F(1,32)=12.13, p=$ $.0015]$, with an interaction between training condition and block $[F(6,192)=3.17, p=.006]$, indicating that the benefit of the delayed starting procedure was mainly evident from Session 3.

The improved performance was related to a higher number of trials with a rapid direct escape during Phase 1 [Trials 9-28, latency maximum $3 \mathrm{sec}$, two-way ANOVA: treatment, $F(1,35)=19.22, p=.0001$; starting condition, $F(1,35)=38.07, p=.0001$; no significant interaction), suggesting that the choline treatment and the delayed starting condition both increased the probability that the rats would be attracted by the suspended cue as soon as they started swimming.

Figure 4 shows that all the rats spent a large amount of time swimming under the suspended cue during the probe 
a)

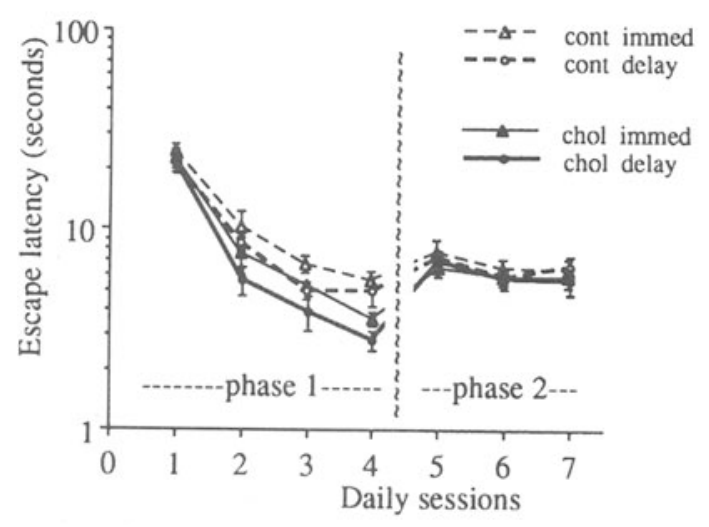

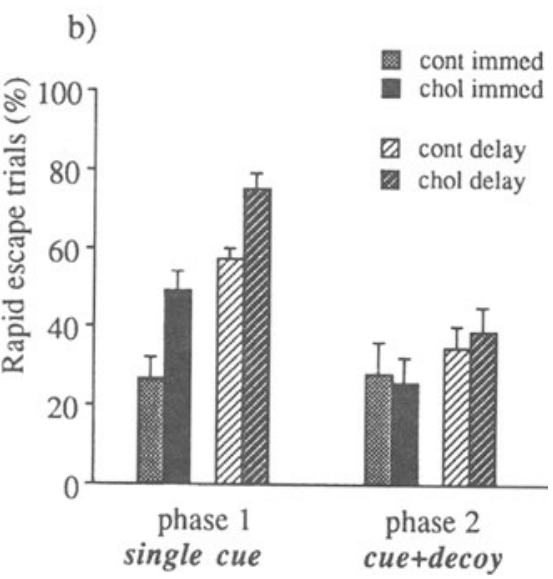

Figure 3. (a) Mean ( $\pm S E M$ ) escape latencies (logarithmic scale) during training on the cued platform at an unpredictable position in space for choline-treated and control rats. Rats trained in the immediate-start condition (immed) were released by hand following the classical procedure; those trained in the delayed-start condition (delay) were allowed to wait for $15 \mathrm{sec}$ on the starting platform at water level before being immersed. Sessions $1,2,3,5$, and 6 consisted of eight trials; Sessions 4 and 7 consisted of four trials (see behavioral procedure). (b) Mean relative frequency of rapid escape trials (latency $\max =3 \mathrm{sec}$ ) during Trials $9-28$ in Phase 1 and during Trials $29-48$ in Phase 2.

trial without platform. A three-way ANOVA (treatment, start condition, and sex) revealed a significant interaction between the treatment and condition effects $[F(1,31)=17.73, p=.0002]$. As for escape latencies, there was no significant effect of sex or interaction with other factors. Separate analyses indicated that the control rats trained in the delayed-start condition spent significantly more time swimming under the suspended cue than did the rats that were directly introduced into the water (Fisher test, significant, $p<.05$ ). In contrast, the choline-treated rats spent a higher amount of time in this area than did the controls only if they were trained in the immediate-start condition (Fisher test, significant, $p<$ .05 ). When they had been trained with a delayed start, they spent less time in the cue area (Fisher test, $p<.05$ ). There was no significant effect of sex in this measure.

Addition of a decoy cue (Phase 2). Figure 3 b shows that all the groups were slower to escape when the decoy cue was added [three-way ANOVA: treatment, condition, and Trials $25-28$ vs. Trials $29-32, F(1,37)=25.80$, $p=.0001]$. During this second training phase, there was a slow decrease in escape latencies $[F(4,148)=2.67$, $p=.03$ ], but no effect of either choline treatment or starting procedure. In fact, there was a very low frequency of trials with a rapid escape $(<4 \mathrm{sec})$ in all the groups during this phase, and both the treatment and the condition effects were nonsignificant.

\section{Discussion}

This experiment demonstrated that the choline treatment facilitated the acquisition of a nonspatial cued task, but did not prevent the distracting effect of an additional irrelevant cue, and did not affect the acquisition of discrimination during the second phase. The use of a starting platform increased the probability that all the rats would detect the position of the cue early in the trial.
This resulted in a higher frequency of rapid escapes and, in the control rats, it increased the time spent swimming under the cue during the probe trial. This second effect might have been due to the fact that the early reinforcements during the training trials increased the attraction of the cue. But the reverse tendency was observed in the choline-treated group in the same condition, suggesting that an early reinforcement does not systematically increase the attraction of the cue. Examination of the paths followed during the probe trials by the choline-treated rats suggested that they tended to alternate between the starting position and that of the suspended cue, whereas the control rats were more concentrated under the cue. This tendency is likely to result in a reduction of the time spent below the cue.

In spite of the positive effect of the starting platform during the acquisition phase, all of the groups were

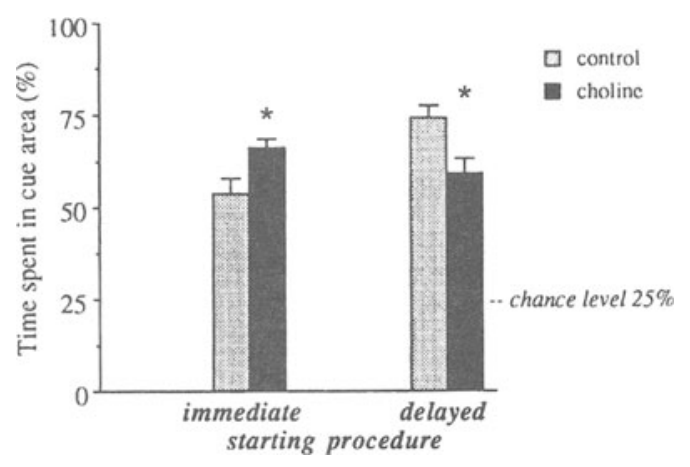

Figure 4. Mean time $\pm S E M$ spent in the training quadrant during a 60-sec probe trial without platform, but with the proximal cue present, for choline-treated and control rats trained either in the delayed-start or in the immediate-start condition. *Significant difference between the choline-treated and control groups. 
equally impaired when the second cue was added, which indicates that the delay did not lead to a better discrimination of the relevant cue. A closer examination of the experimental design suggests, however, that this task was in fact quite difficult. It can be considered as a simple nonspatial discrimination task because the position of the platform was randomly determined, and the rats had already been trained to approach the relevant cue during Phase 1 . However, the $60-\mathrm{sec}$ probe trial during which they were left for $60 \mathrm{sec}$ in the pool in the absence of the escape platform might have served as an extinction trial. Moreover, only four different spatial positions were used, which the rats might have learned as well, and the distractor cue was necessarily on one of these positions. The fact that the latency was also increased in the groups with a delayed start suggests that the attraction of the decoy cue was equally strong when the rats were allowed more time to discriminate among the cues. This might explain why the performance remained very low during the second phase, which was also obviously too short to allow for an accurate discrimination of the correct cue in both conditions.

The fact that the treated rats were more rapid in reaching the cued platform confirms that the improvement observed in Experiment 1 was related to a more efficient use of the suspended cue. However, the deleterious effect of the decoy indicates that all the groups were equally disturbed by the additional irrelevant marker, and that neither the presence of a starting platform nor the choline treatment led to better discrimination.

\section{EXPERIMENT 3}

The experiments conducted with the Morris task have revealed that the choline-treated rats were more rapid to detect and to swim toward a salient cue. But the treated subjects showed only little evidence of directly improved spatial abilities. Experiment 3 was designed to assess spatial memory on solid ground, using a task that was sensitive to the improvement due to the pre- or posttraining treatment with a nootropic drug (Schenk \& Gafner, 1993) and to aging deficits (Schenk et al., 1990). All of the rats were trained in a place-learning task in the homing arena (Schenk, 1989). Since it is a dry-land task and the rats are gently deposited at the starting positions, they should have enough time to orient toward the goal sector, a condition that is similar to that of the delayed starting procedure in Experiment 2. Only a few training trials were given, so as to make the task more difficult for the normal rats. A probe trial was conducted early in training, after removal of the goal cue. Further training was conducted with a pure place-learning design. All the rats were then trained to learn a new position, and retention was tested following a 24 -h interruption.

The rats used in this experiment were tested at the age of 7 months (i.e., 5 months after treatment cessation, which also provides information about its long-term effect).

\section{Method}

Subjects and treatment. Twenty-two control rats (11 males and 11 females) and 22 choline-treated rats ( 11 males and $11 \mathrm{fe-}$ males) tested in the previous experiment were used. They were maintained as described above.

Apparatus. The apparatus used for this experiment was a large circular homing arena $($ diam $=160 \mathrm{~cm})$ enclosed by $40-\mathrm{cm}$-high walls, described elsewhere (Schenk, 1989; Schenk et al., 1990). It was provided with 15 possible escape holes arranged in 5 groups of 3, as shown in Figure 5. Only one hole allowed passage to the subject's home cage placed under the table. All the holes were covered by a disk of white plastic of the same material as the sheet covering the arena floor. The room was rich in distal cues, and the table was indirectly lit by four 60 -watt bulbs. A camera was placed directly above the center of the table.

Behavioral procedure. For a training trial, the rat was deposited in one of 5 possible start locations facing the center of the arena. For the first 3 trials, the rats were allowed up to $3 \mathrm{~min}$ to find and enter the escape hole. Those not finding the connected hole in the allotted time were gently placed on it. For the first trials, the holes remained partially uncovered, and they were progressively masked during the following trials, and were completely covered by Trial 7 . The table was rotated after each trial, and the cover and metal fitting of the hole through which the rat had entered were changed, so that no relevant olfactory trace might remain attached to the training position.

All of the rats were trained to find the escape hole at a fixed position according to distant room cues. For the first 10 trials (Days 1 and 2), a salient cue was placed near the escape hole. This cue was absent during the third daily training session (Trials 12-16). Two $72-\mathrm{sec}$ probe trials with no hole connected and no proximal cue were conducted at the end of the second and third daily sessions.

On the next day, 14 females ( 6 control and 8 choline-treated rats) were given a new probe trial (extinction), at the end of which they were allowed to escape from the table at a new position in space. They then received 5 additional trials to learn this new position in the absence of any proximal cue. Twenty-four hours later, they were given a last probe trial with no hole connected.

All of the trials were video recorded. Escape latency (i.e., the latency to uncover the escape hole) was recorded during the training trials. The probe trials were analyzed by the XY video tracker, and the time spent in the vicinity of the 5 groups of 3 holes was recorded for $72 \mathrm{sec}$.

\section{Results}

Phase 1. All of the rats learned to escape through the connected hole. Figure 6a shows that during training with the proximal cue present, the escape latency (i.e., the time spent before removing the plastic cover on the escape hole) decreased to reach a stable value [three-way ANOVA: treatment, sex, and Trials 1-10-trial effect, $F(9,360)=4.33, p=.0001]$. No effect of treatment or sex was evident during this period. During Session 3, while training was continued without the proximal cue, the latency increased, most likely because of the removal of the cue [three-way ANOVA: Session 2 vs. Session 3, $F(1,40)=10.93, p=.002$; no significant effect of treatment or sex]. The choline-treated rats had shorter escape latencies than the control rats during Session 3 only [three-way ANOVA: treatment, sex, and Trials 11-15treatment effect, $F(1,40)=4.71, p=.036$ ]. There was no significant change during this session, nor was there any difference between the female and male groups. 

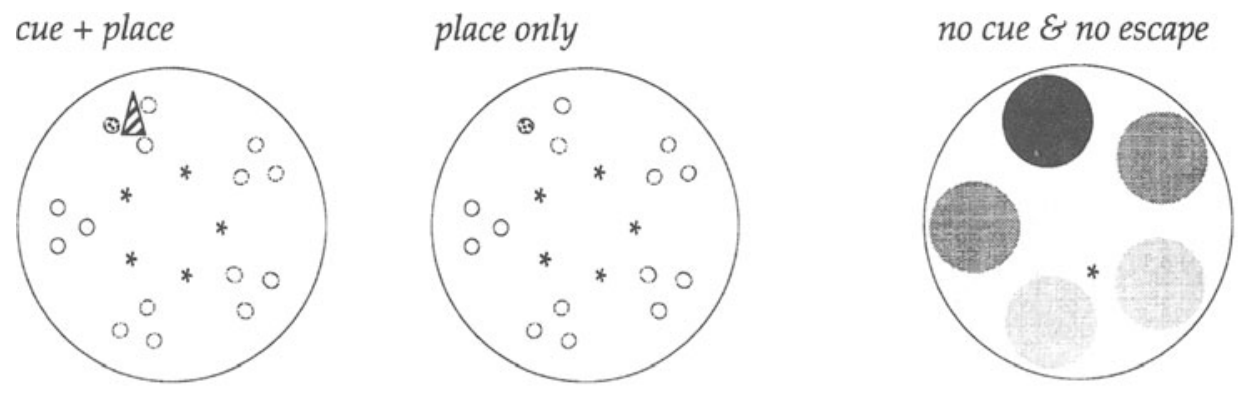

training trials $(1-10,12-16)$

probe trials $(11,17)$

connected hole
d local landmark
* start positions

hole areas

Figure 5. Schematic representation of the homing arena with the starting positions $\left({ }^{*}\right)$, the position of the cue (local landmark), and the surface of the hole areas. During the training trials, escape-path length was measured as the distance walked between the starting position and entrance into the training-hole area. For the probe trials, the time spent in each hole area was recorded for 72 sec. (From Schenk \& Gafner, 1993.)

Individual escape paths were measured in Sessions 2 and 3 as the distance crossed between the start and the training-hole area. Figure $6 \mathrm{~b}$ shows that the cholinetreated rats followed shorter paths than the control rats in both sessions. A three-way ANOVA confirmed a significant effect of treatment $[F(1,40)=15.79, p=.0003]$ and cue removal [session effect, $F(1,40)=36,82, p=$ $.0001]$, but no effect of $\operatorname{sex}[F(1,40)=.38$, n.s.], nor any interactions. The treatment effect was highly significant in Session 2 [one-way ANOVA; $F(1,42)=12.92, p=$ $.0008]$ and Session $3[F(1,42)=11.60, p=.002]$. Thus, in Session 2, the path followed by the treated rats to reach the training area was significantly shorter, while the latency to uncover the escape hole was comparable to that of untreated rats. This is related to the fact that most of the rats did not uncover the training hole as soon as they entered the training area. Actually, $23 \%$ of the controls and $22 \%$ of the choline-treated rats left this area for an exploratory bout before uncovering the hole.

During the early probe trial conducted at the end of the second daily session, there was a general discrimination of the training sector [three-way ANOVA, $F(4,160)=$ $5.45, p=.004$ ] but no effect of sex or treatment, in spite of the fact that the sector effect was evident in the two female groups only. During the probe trial conducted at the end of Session 3, there was a highly significant discrim-
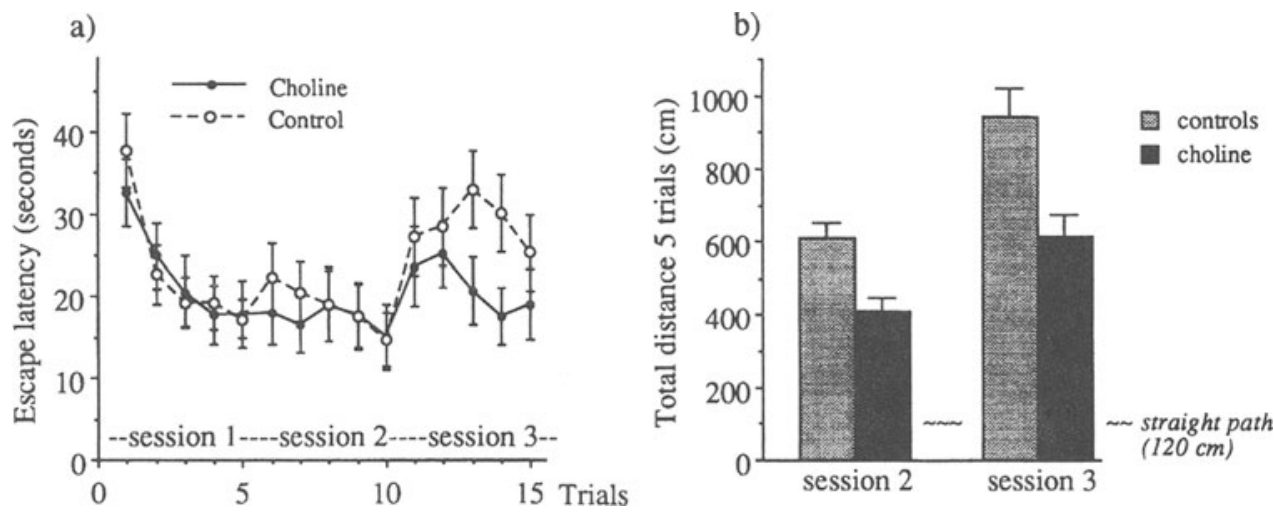

Figure 6. (a) Mean escape latencies ( $\pm S E M$ ) during training in the homing arena for choline-treated and control rats. The proximal cue was placed in the center of the training sector during Sessions 1 and 2 , and was absent during Session 3. (b) Mean distance to enter the training-hole area during Sessions 2 and 3 in the control and choline-treated groups. 

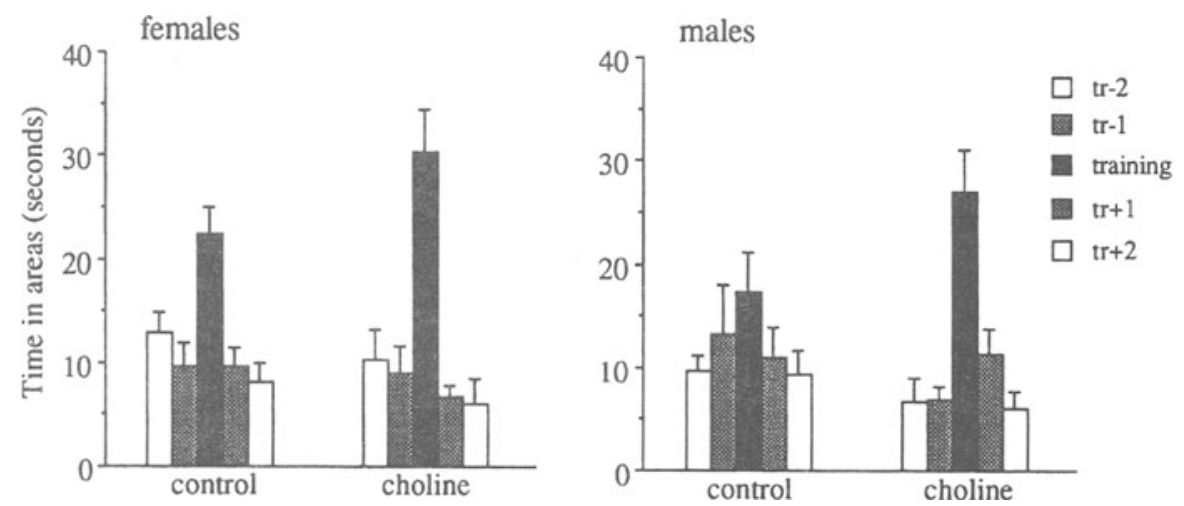

Figure 7. Mean time ( $\pm S E M$ ) spent in the five hole areas during the second probe trial (end of Session 3), with no connected hole and no proximal cue present. The different hole areas are identified on the basis of their position relative to that of the training hole (training), either adjacent right or left $(t r+1, \operatorname{tr}-1)$ or opposite right or left $(\operatorname{tr}+2, \operatorname{tr}-2)$.

ination of the training sector $[F(4,160)=21.62, p=$ $.0001]$, together with a significant interaction between sector discrimination and treatment $[F(4,160)=3.14$, $p=.016]$. This was due to a significant effect of treatment on the relative time spent in the training sector $[F(1,40)=6.75, p=.013]$ with no sex effect $[F(1,40)=$ .37], nor any interaction between sex and treatment. Figure 7 shows the bias toward the sector around the training hole. Separate one-way ANOVAs of the time spent in the five sectors by each group revealed that the group of male controls did not show a significant bias toward the training sector, whereas this effect was highly significant in the female controls and in the two choline-treated groups $\left[F_{\mathrm{S}}(4,40)\right.$, significant, $\left.p<.005\right]$.

Phase 2. The last probe trial was conducted $24 \mathrm{~h}$ after a five-trial session during which the rats were trained to find the escape hole at a new position. A two-way ANOVA showed no significant bias toward the new training sector nor any treatment effect.

\section{Discussion}

This experiment showed that the choline-treated rats were more direct than control rats in reaching an escape position indicated by a salient cue, although they were no more rapid in uncovering the connected hole. Moreover, they showed a better discrimination of the training position after the salient cue had been removed. There was no interaction between the drug treatment and sex effect. No group showed a significant discrimination of a new uncued training sector $24 \mathrm{~h}$ after training with this new position.

These results confirm the results obtained in the Morris navigation task and indicate that following removal of the goal cue, the treated rats were not only more inclined to search on the correct spot, they were also more efficient in reaching this position from any starting area.

\section{GENERAL DISCUSSION}

The present results demonstrate that supplemental dietary choline given perinatally and postnatally until the age of 2 months can improve the performance measured in a place-navigation task and in a dry-land version of this task up to 4 months after cessation of the daily supplementation. The behavioral changes were similar in males and in females, but were only partial in juvenile subjects. This effect was very selective and showed three specific features.

First, improved acquisition was systematically observed in all three experiments when the rats learned to reach a goal signaled by a salient cue. When the goal was at a fixed position in space, the improvement was similar in the water and the dry-land tasks. It was also significant in a nonspatial design in the Morris water maze, whether early orientation toward the cue was facilitated by a delayed starting procedure or not. But the treatment did not prevent the distracting effect of adding a secondary irrelevant cue during training.

Second, the facilitation observed in the cued conditions persisted in both tasks after removal of the cue. In the water task, there was enhanced searching in the correct position during the probe trial. In the dry-land task, the treated rats were more efficient in reaching the escape area and also showed a stronger bias toward the training position. When the task was nonspatial, the treated rats were not more attracted systematically by the goal cue during the probe trials than were the control rats. In juvenile rats, which are known to express poor spatial bias in a place-learning task (Schenk, 1985; Schenk 1989), the positive effect of the treatment on escape performance was not accompanied by an increased spatial bias.

Third, the effects on spatial memory were mainly indirect and secondary to a previous training phase in the presence of the goal cue. Indeed, the choline treatment 
affected path length very little, at the end of the acquisition in the place-only condition in the Morris maze, and there was no effect on the spatial bias in probe trials conducted following place training.

Thus, although very selective, these effects do not seem to be due to a specific improvement in spatial abilities, and it is tempting to explain the enhanced performance of the treated rats by mere enhanced swim speed, motivation, or reactivity. But a comparison of the behavior in different situations suggests that it is not the case. First, the reduced latencies were systematically related to a decrease in path length, indicating that the treatment effect was not merely one of increasing swim speed. Moreover, it is not clear why a change in speed would be more likely in the presence of a salient cue, or why this would be accompanied by a more pronounced spatial bias following removal of the cue. Second, if the improvement was secondary to an overall increase in escape motivation, it should not depend on the presence of the cuc. The hypothesis of an increased motivation to escape from the arena seems also to contradict the fact that, in the homing task, the treated rats were no more rapid to uncover the escape hole than the controls. Finally, the hypothesis that the treated rats were simply hyperreactive toward a salient cue does not account for the observed effects. Since the reactivity of the treated rats toward the goal cue was accompanied by an increased spatial bias after removal of this cue, they were at the same time more attentive to the landnarks around the experimental enclosures.

To account for the fact that the treatment improved performance in apparently the easiest cued training condition, it is necessary to consider why an optimal level was not reached by the control subjects. A closer look at the experimental situation suggests that in this condition, a mere visual guidance could not lead to an optimal performance, as it would if the platform were simply visible. Indeed, the black hanging cylinder appears very salient from the start areas. But when the rats arrive in the vicinity of the platform, they cannot reach the cue that is hanging above them and that also appears less visible, as the cylinder (diam $=4.5 \mathrm{~cm}$ ) is then seen from one end. A cue guidance thus offers only partial help, as the final movement to land on the platform must be adjusted relative to distant room cues. There was a similar difficulty in the homing arena, as the cue was in the center of a group of 3 holes, only one of which was connected. Thus, each of these cued tasks required an optimal combination of cue guidance and place memory. The hypothesis that the task cannot be solved by a simple guidance strategy is confirmed by the significant deficit shown by rats with medial septum lesions in this training condition (Brandner, Berchtold, \& Schenk, 1994). The lesioned rats showed accurate swim trajectories in arriving in the vicinity of the platform, but followed longer correction paths when they missed the platform. The difficulty of the task might thus be related to the fact that it requires the linking of two different strate- gies that are not readily compatible with one another. Indeed, as discussed by Jaffard and Meunier (1993), an optimal behavior might require an active process "that readily upsets the imbalance between competing memory systems" (p. 211).

With reference to the conditioning theories (Dickinson, 1980), one can consider that the presence of a salient local cue might overshadow the distant cues outside the pool. In accord with this hypothesis, rats that have been trained to escape onto a visible platform showed a low spatial bias toward the training quadrant during a probe trial without a platform (Morris, 1981). In Experiment 1, the youngest rats showed a poor bias toward the training position following removal of the cue (see Figure 2), which indicates an important overshadowing effect in immature subjects and confirms our earlier findings (Schenk, 1985; Schenk et al., 1995). The effect of cue removal was also significant, but much less important, in the adult control group. This suggests that the main effect of the choline treatment in adult rats can be described as facilitating an optimal behavior combining cue guidance with a place strategy. Although hypothetical, this interpretation might account for the very specific advantage observed in cued training.

With a similar, though less prolonged, choline treatment, Meck et al. (1989) found an improvement in reference and working spatial memory in a radial-maze task. This improvement was maintained in aged rats and it was associated with a lower rate of forgetting in interruption trials with a delay (Meck \& Williams, 1992). Moreover, Dallal et al. (1992) showed a selective effect of choline supplementation that resulted in advancing the appearance of place navigation in juvenile rats, but they did not find any effect on cued navigation. This seems to indicate a more general influence of the treatment on spatial memory. In our experiments, however, the lack of effect on place learning suggests that the improvement of spatial memory was only secondary to a facilitation in the development of appropriate approach strategies in the presence of a salient cue. In addition, although we found similar effects of the treatment in males and in females, a choline supplementation has elsewhere appeared to be less effective in improving performance in female rats (Loy et al., 1992), and it has been shown that the presence of male steroids during the neonatal period plays a critical role in the expression of memory improvement and of hippocampal NGF increases (Gorry, Loy, Blusztajn, Meck, \& Williams, 1992). These discrepancies might in part be due to a different treatment, to different cuing procedures, and to the use of different strains of rats (these authors used Sprague-Dawley albino rats, whereas we used hooded PVG rats).

A pilot experiment conducted in a radial-maze task after completion of Experiment 3 confirmed that our choline-treated rats tended to be more accurate in this task. Facilitating the identification of the relevant configuration of distal cues that characterizes each arm might improve accuracy in the radial-maze task, even 
though efficient patrolling by normal rats is certainly not exclusively based on the identification of armassociated cues (Suzuki, Augerinos, \& Black, 1980). However, it is not clear whether radial-maze and placelearning tasks have the same requirement in spatialmemory capacity. It is not clear, either, what specific role is played by the cholinergic system in spatial memory. As mentioned in the introduction, the forebrain cholinergic projection system does not appear to play an equally critical role in all the place-learning experiments conducted with the Morris navigation task (see, e.g., Sinden et al., 1995). Rats' performance may appear significantly impaired (Hagan, Salamone, Simpson, Iversen, \& Morris, 1988), only marginally impaired (McAlonan, Wilkinson, Robbins, \& Everitt, 1995), or even not impaired at all (Decker et al., 1992) following excitotoxic lesion of the medial septal nucleus. But the same lesioned rats were selectively impaired in an experimental design for place discrimination, as if disturbed by the presence of two salient cues (Decker, Radek, et al., 1992; Decker, Majchrzak, \& Anderson, 1992). Moreover, we have found that rats with quisqualic lesions of the medial septal nucleus remained unimpaired in place learning in the Morris task but were considerably impaired when trained with the hanging cue (Brandner et al., 1994). A logical interpretation might be that this apparent variability results from a poor reliability in the damage to the cholinergic system, but the same individuals were unimpaired in the water place-learning task and very poor in the dry-land task with a cued escape position like that used in Experiment 3 (Schenk et al., 1995). Thus, damage to the septohippocampal cholinergic fibers does not prevent all forms of spatial learning, although it is not yet clear which type of task is affected or why. Why cued spatial tasks can be selectively affected must thus be further elucidated.

More generally, an optimal tuning of the cholinergic system appears indispensable for efficient spatial learning. But there are diverse interpretations as to the function of cholinergic activation for solving spatial tasks. Cholinergic blockade with muscarinic antagonists impairs various components of spatial abilities such as the sensitivity to distant cues (Hagan, Tweedie, \& Morris, 1986; Whishaw, 1989; Whishaw \& Petrie, 1988); the organization of exploratory responses (Buhot, Soffié, \& Poucet, 1989; Whishaw \& Tomie, 1987), or the development of appropriate behavioral strategies necessary for the acquisition of movement sequences under distal-cue guidance (Whishaw \& Petrie, 1988). Since cholinergic blockade acted mainly when administered before training (see Aigner, Walker, \& Mishkin, 1991; Hagan et al., 1986), this treatment might interfere with the initial storage of information, or with the process by which ongoing information is integrated before the selection of an appropriate behavior or strategy. Along this line, recent experiments have shown that a cholinergic dysfunction does affect the attention to environmental stimuli (Callahan, Kinsora, Harbaugh, Reeder, \& Davis, 1993; Muir,
Dunnett, Robbins \& Everitt, 1992). This suggests an involvement in attentional processes and in the selection of an appropriate strategy as well, which does not necessarily exclude a participation in memory processes.

In conclusion, a choline supplementation is likely to have a much broader effect on brain development than just the specific cholinergic changes reviewed above. Moreover, this uncertainty is amplified by the poverty of adequate psychological components to match with the supposed function of a given neural process. Although a dissociative approach confirms that spatial memory is primarily affected by lesions of the hippocampal region and might thus represent a unitary process, it does also reveal three different components of spatial abilities. Remaining in a place that had been positively reinforced, approaching a cue predicting the availability of a reinforcement, and patrolling on the basis of a spatial representation each depends on the participation of a different brain structure - respectively, the amygdala, the striatum, and the hippocampus (McDonald \& White, 1993). Spatial behavior is thus not a unitary process, and improvements in spatial tasks might not be exclusively due to an increased capacity to integrate spatial information. Only detailed behavioral analyses will be able to resolve this issue.

\section{REFERENCES}

Aigner, T. G., WALKer, D. L., \& Mishkin, M. (1991). Comparison of the effects of scopolamine administered before and after acquisition in a test of visual recognition memory in monkeys. Behavioral \& Neural Biology, 55, 61-67.

Brandner, C., Berchtold, N., \& Schenk, F. (1994). Cholinergic and noradrenergic modulation in spatial learning and/or attention. European Journal of Neuroscience, 7, 117.

Buhot, M.-C., Soffí́, M., \& Poucet, B. (1989). Scopolamine affects the cognitive processes involved in selective object exploration more than locomotor activity. Psychobiology, 17, 409-417.

Callahan, M. J., Kinsora, J. J., Harbaugh, R. E., Reeder, T. M., \& Davis, R. E. (1993). Continuous infusion of scopolamine impairs sustained attention of rhesus monkeys. Neurobiology of Aging, 14, 147-151.

Chevalley, A.-F., \& Schenk, F. (1987). Immature processes of spatial learning in hooded rats. Society for Neuroscience Abstracts, 13, 184.

Dallal, N. L., Meck, W. H., \& Williams, C. L. (1992). Selective developmental acceleration of relational cue navigation and reduction of age-related impairments in spatial processing by prenatal supplementation with choline. Society for Neuroscience Abstracts, $18,147$.

Decker, M. W., Majchrzak, M. J., \& Anderson, D. J. (1992). Effects of nicotine on spatial memory deficits in rats with septal lesions. Brain Research, 572, 281-285.

Decker, M. W., Radek, R. J., Majchrzak, M. J., \& Anderson, D. J. (1992). Differential effects of medial septal lesions on spatialmemory tasks. Psychobiology, 20, 9-17.

Dickinson, A. (1980). Contemporary animal learning theory. Cambridge: Cambridge University Press.

Gorry, E., Loy, R., Blusztajn, J. K., Meck, W. H., \& Williams, C. L. (1992). Gonadal steroids and choline interact during development to improve radial-arm maze performance and increase hippocampal NGF in adult rats. Society for Neuroscience Abstracts, $18,147$.

Hagan, J. J., Salamone, J. D., Simpson, J., Iversen, S. D., \& Morris, R. G. M. (1988). Place navigation in rats is impaired by lesions of medial septum and diagonal band, but not nucleus basalis magnocellularis. Behavioural Brain Research, 27, 9-20.

Hagan, J. J., Tweedie, F., \& Morris, R. G. M. (1986). Lack of task 
specificity and absence of posttraining effects of atropine on learning. Behavioral Neuroscience, 100, 483-493.

Jackson, D. A., Meck, W. H., Williams, C. L., \& Blusztajn, J. K. (1992). Supplementation with choline in utero reduces brain choline acetyltransferase (CAT) in the rat. Society for Neuroscience $\mathrm{Ab}$ stracts, $18,147$.

JAFFARD, R., \& MEUnieR, M. (1993). Role of the hippocampal formation in learning and memory. Hippocampus, 3, 203-218.

Loy, R., Heyer, D., Miller, J., \& Linder, M. D. (1992). Sex differences in the effect of prenatal choline treatment on septal cell size and hippocampal NGF. Societv for Neuroscience Abstracts, 18, 548.

Loy, R., Heyer, D., Williams, C. L., \& Meck, W.H. (1991). Cholineinduced spatial memory facilitation correlates with altered distribution and morphology of septal neurons. In T. C. Napier, P. W. Kalivas, \& I. Hanin (Eds.), The basal forebrain (pp. 373-382). New York: Plenum.

McAlonan, G. M., Wilkinson, L. S., RobBins, T. W., \& Everitt, B. J. (1995). The effects of AMPA-induced lesions of the septohippocampal cholinergic projection on aversive conditioning to explicit and contextual cues and spatial learning in the water maze. European Journal of Neuroscience, 7, 281-292.

MCDonald, R. J., \& White, N. M. (1993). A triple dissociation of memory systems: Hippocampus, amygdala, and dorsal striatum. Behavioral Neuroscience, 107, 3-22.

Meck, W. H., Smith, R. A., \& Williams, C. L. ( 1989). Organizational changes in cholinergic activity and enhanced visuospatial memory as a function of choline administered prenatally or postnatally or both. Behavioral Neuroscience, 103, 1234-1241.

MeCK, W. H., \& Williams, C. L. (1992). Choline supplementation during prenatal development inoculates against the age-related decline in spatial memory of 24-26 month old rats. Society for Neuroscience Ahstracts, $18,147$.

Morris, R. G. M. (1981). Spatial localization does not require the presence of local cues. Learning \& Motivation, 12, 239-260.

Muir, J. L., Dunnett, S. B., Robiins, T. W., \& Everitt, B. J. (1992). Attentional functions of the forebrain cholinergic systems: Effects of intraventricular hemicholinium. physostigmine, basal forebrain lesions and intracortical grafts on a multiple-choice serial reaction time task. Experimental Brain Research, 89, 611-622.

SCHENK, F. (1985). Development of place navigation in rats from weaning to puberty. Behavioral \& Neural Biology, 43, 69-85.

SCHENK, F. (1989). A homing procedure for studying spatial memory in immature and adult rodents. Journal of Neuroscience Methods, 26, 249-258.

Schenk, F. , Contant, B., \& Werffeli, P. (1990). Intrahippocampal cholinergic grafts in aged rats compensate impairments in a radial maze and in a place learning task. Experimental Brain Research, 82, 641-650

Schenk, F., \& Gafner, M. (1993). Eine akute Piracetambehandlung verbessert die Ortserkennung in jungen weiblichen Ratten. In P. Baumann (Ed.), Biologische Psychiatrie der Gegenwart (pp. 778781). Vienna: Springer-Verlag.

Schenk, F., Grobéty, M.-C., Lavenex, P., \& LipP. H. P. (1995). Dissociation between basic components of spatial memory in rats. In E. Alleva, A. Fasolo, H-P. Lipp, L. Nadel, \& L. Ricceri (Eds.), Behavioral brain research in naturalistic and semi-naturalistic settings: Possibilities and perspectives (pp. 277-300). Dordrecht: Kluwer.

Sinden, J. D., Hodges, H., \& GRAY, J. A. (1995). Neural transplant and recovery of cognitive function. Behavioural \& Brain Sciences, 18, 10-35.

SNEDECOR, G. W., \& COCHRAN, W. G. (1971). Statistical methods (6th ed.). Ames: lowa State University Press.

Suzuki, S., Augerinos, G., \& Black, A. H. (1980). Stimulus control of spatial behavior on the eight-arm maze in rats. Learning \& Motivation, $11,1-18$

TEEs, R. C., \& JoHnSton, J. L. (1992). Effect of pre- and/or postnatal choline supplementation on working and reference memory (spatial and nonspatial) and open field behavior in the rat. Society for Neuroscience Abstracts, 18, 548 .

Whishaw, I. Q. (1989). Dissociating performance and learning deficits on spatial navigation tasks in rats subjected to cholinergic muscarinic blockade. Brain Research Bulletin, 23, 347-358.

Whishaw, I. Q., \& Petrie, B. F. (1988). Cholinergic blockade in the rat impairs strategy selection but not learning and retention of nonspatial visual dicrimination problems in a swimming pool. Behavioral Neuroscience, 120, 662-677.

Whishaw, I. Q., \& TomiE, J.-A. (1987). Cholinergic receptor blockade produces impairments in a sensorimotor subsystem for place navigation in the rat: Evidence from sensory, motor, and acquisition tests in a swimming pool. Behavioral Neuroscience, 101, 603-616.

(Manuscript received January 5, 1994; revision accepted for publication May 19, 1995. . 